\title{
Assessment of the Surgical Workforce Pertaining to Cytoreductive Surgery and Hyperthermic Intraperitoneal Chemotherapy in the United States
}

\author{
Darryl Schuitevoerder, MBBS ${ }^{1}$, Scott K. Sherman, MD ${ }^{1}$, Francisco J. Izquierdo, MD ${ }^{1,2}$, Oliver S. Eng, MD ${ }^{1}$, and \\ Kiran K. Turaga, MD, MPH ${ }^{1}$ \\ ${ }^{1}$ Department of Surgery, Section of General Surgery and Surgical Oncology, University of Chicago, Chicago, IL; \\ ${ }^{2}$ Department of Surgery, Clinica Santa Maria, Santiago, Chile
}

The incidence of peritoneal metastases varies widely depending on the primary tumor site, with estimates ranging from 8 to $17 \%$ for colorectal cancers and $14 \%$ to $20 \%$ for gastric cancers. The estimate for ovarian cancers is $60 \%{ }^{1-3}$ Data regarding the optimal management of peritoneal metastases from non-gynecologic malignancies have been limited due to the exclusion of patients with peritoneal metastases from clinical trials. ${ }^{4}$ However, studies have shown that cytoreductive surgery (CRS) and hyperthermic intraperitoneal chemotherapy (HIPEC) improve survival for well-selected patients with peritoneal metastases. ${ }^{5,6}$

The true incidence of patients eligible for CRS/HIPEC is difficult to ascertain but is estimated to be 29,000 to 41,000 per year in the United States. ${ }^{7}$ Interest in this field continues to grow, with the number of active clinical trials involving CRS/HIPEC totaling more than four times that of previously published clinical trials in this field. ${ }^{8}$ Concurrently, the number of publications pertaining to CRS and HIPEC indexed in PubMed has increased from respectively 98 and 648 in 2000 to 720 and 1541 in 2019.

As interest in the benefits of CRS/HIPEC has grown, many institutions have established new CRS/HIPEC treatment programs, often tasking these procedures to recent graduates. However, CRS/HIPEC is a complex time-

(C) Society of Surgical Oncology 2020

First Received: 18 November 2019; Published Online: 6 July 2020

K. K. Turaga, MD, MPH

e-mail:kturaga@surgery.bsd.uchicago.edu and resource-intensive oncologic surgical procedure requiring significant individual and institutional expertise to overcome the technical and oncologic learning curve, which involves performing respectively 130 to 220 cases $^{7,9-12}$ and 90 cases. $^{11}$

The recent growth and interest in this field have required that the pool of surgeons performing CRS/HIPEC expand to meet the demand. Little is known about the current workforce performing particular procedures in the United States, and to our knowledge, no studies or analyses are detailing the surgical workforce performing pancreaticoduodenectomy, major hepatectomy, esophagectomy, or CRS/HIPEC.

Unlike the aforementioned similarly complex oncologic procedures ${ }^{13} \mathrm{CRS} / \mathrm{HIPEC}$ has no training requirements and no requisite experience in either residency or fellowship. Without a thorough understanding of the disease processes, the true complexity of CRS/HIPEC may be underestimated, and some surgeons performing these procedures may lack the requisite experience and support to achieve adequate oncologic outcomes while maintaining patient safety.

To understand the imbalance that currently exists between the experience of surgeons performing CRS/ HIPEC and established data regarding the learning curve for these procedures, a better definition of the current workforce performing CRS/HIPEC is needed. Thus, we sought to determine the characteristics of surgeons and programs currently performing CRS/HIPEC in the United States. 


\section{METHODS}

\section{Identification of the Cohort}

To identify CRS/HIPEC surgeons, we used a hierarchical systematic Internet search strategy. An institutionlevel search was directed at the list of Association of American Medical Colleges (AAMC)-affiliated medical schools and National Cancer Institute (NCI)-designated cancer centers. An industry-level search was performed using a commercially maintained database at hipectreatment.com. Patient-level sources were used from PMPpals.net. A hand-search strategy using Google (Mountain View, CA) systematically combined each state with the term "HIPEC" to identify physicians and programs that might have been missed.

For each physician, we recorded current institution, specialty and subspecialty training, and years removed from training. For surgeons without a listed date of training completion, we checked board certification status through the American Board of Surgery website, extrapolating the date of training completion from this by adding the number of years expected for completion of their fellowship (i.e., 2 years for surgical oncology) to the date of initial board certification.

The number of CRS/HIPEC surgeons per area was obtained from the United States Census Bureau. ${ }^{14,15}$

\section{Learning Curve Data}

Data regarding the number of cases required to meet the operative inflection point were obtained from prior studies examining either a consecutive cohort of patients or adjusted models to establish the learning curve for CRS/ HIPEC. $^{7,9-12}$ To frame and provide the context necessary for the discussion surrounding learning curves for CRS/ HIPEC, Rajeev et al. $^{7}$ estimated the average volume of cases per year at several centers known for their CRS/ HIPEC programs and found that in 2016, the average requisite number of cases was 55 (range, 24-123) per year.

\section{RESULTS}

\section{Institution and State-Specific Data}

The findings showed that CRS/HIPEC is performed at 121 institutions by a mean of 1.6 surgeons (interquartile range [IQR], 1-3) per institution. The majority of the centers are academic or academic-affiliated centers (Fig. 1). Of the 50 states, 12 had no surgeons performing CRS/HIPEC (Fig. 2).

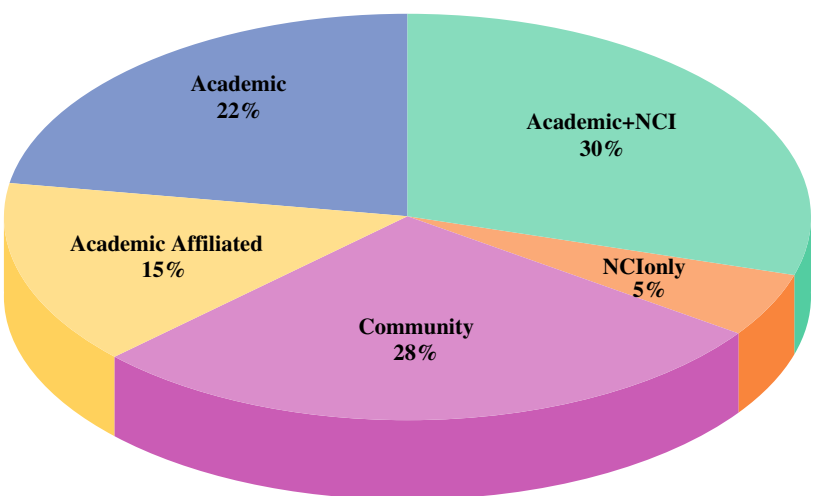

FIG. 1 Centers Performing CRS/HIPEC in the United States. Academic centers are university/medical school based hospitals, while academic affiliated centers are private hospitals affiliated with a university/medical school. NCI National Cancer Institute

The state with the highest number of surgeons performing CRS/HIPEC is New York $(n=21)$, followed by California $(n=13)$, Illinois $(n=12)$, Florida $(n=11)$, Ohio $(n=11)$, and Texas $(n=11)$. Standardization by population showed that the average number of surgeons performing CRS/HIPEC per 10 million persons is 5.69 (IQR, 2.05-8.99). Excluding Washington D.C., with its 68.3 surgeons per 10 million population, Kansas boasts the highest absolute concentration, with 24 surgeons per 10 million persons performing CRS/HIPEC. Among the states with at least one surgeon performing CRS/HIPEC, South Carolina has the lowest concentration of CRS/HIPEC surgeons (1.97 per 10 million persons). Standardization by regions showed that the Midwest has the highest number of HIPEC surgeons per capita (9.1 per 10 million) compared with 8.4 per 10 million in the Northeast, 4.7 per 10 million in the South, and 3.2 per 10 million in the West (Fig. 2).

Standardization by area, excluding Washington D.C., showed that the average number of surgeons performing CRS/HIPEC is 1.21 per 10,000 square miles (IQR, $0.072-1.21$ square miles). Of the major U.S. regions, the Midwest has the highest concentration of CRS/HIPEC surgeons, with 3.4 CRS/HIPEC surgeons per 10,000 square miles, whereas the West has the lowest concentration, with 0.13 CRS/HIPEC surgeons per 10,000 square miles.

\section{Surgeon-Specific Data}

Our search identified 193 surgeons currently performing CRS/HIPEC in the United States. Of the surgeons performing CRS/HIPEC, the majority are fellowship-trained in surgical oncology $(76 \%), 11 \%$ are gynecologic oncologists, $4 \%$ have completed hepatopancreatobiliary (HPB) training, 3\% have completed colorectal training, $4 \%$ have completed dual-fellowship training, and $4 \%$ have completed general surgery residency without further 


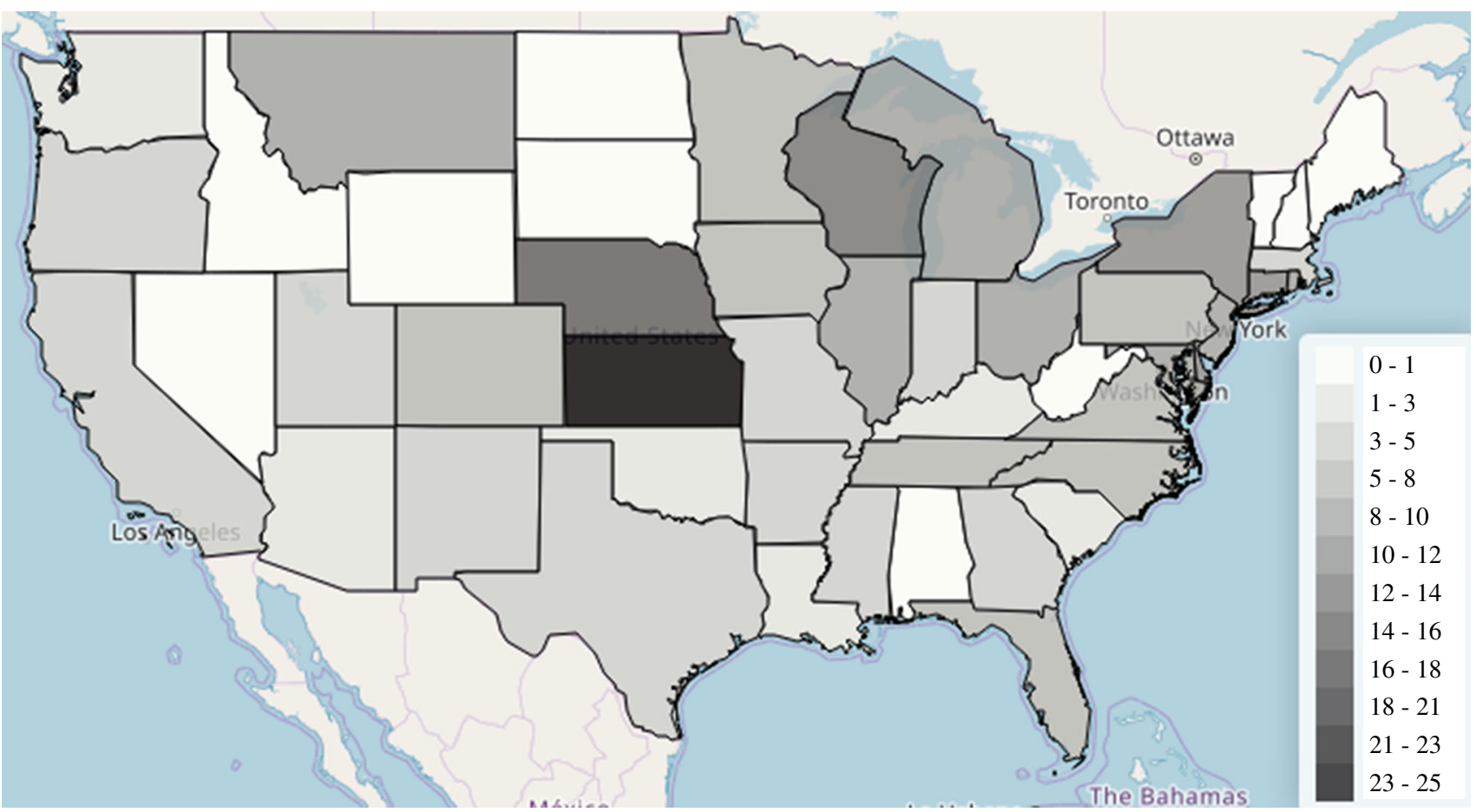

FIG. 2 Heat map of surgeons performing CRS/HIPEC in the United States. Legend represents number of surgeons per 10 million persons in each of the following states: Alabama, Alaska (not shown), Hawaii (not shown), Idaho, Maine, Nevada, New Hampshire, North Dakota,

subspecialty training. Those with dual-fellowship training have completed a surgical oncology fellowship in addition to one of the following: surgical critical care, HPB, colorectal surgery, transplant, or minimally invasive surgery.

The median time removed training was 11 years (IQR, $5-15.25$ years). The majority of surgeons had been practicing independently for 1 to 15 years, and a few had been practicing 30 years or longer (Fig. 3). Among the current

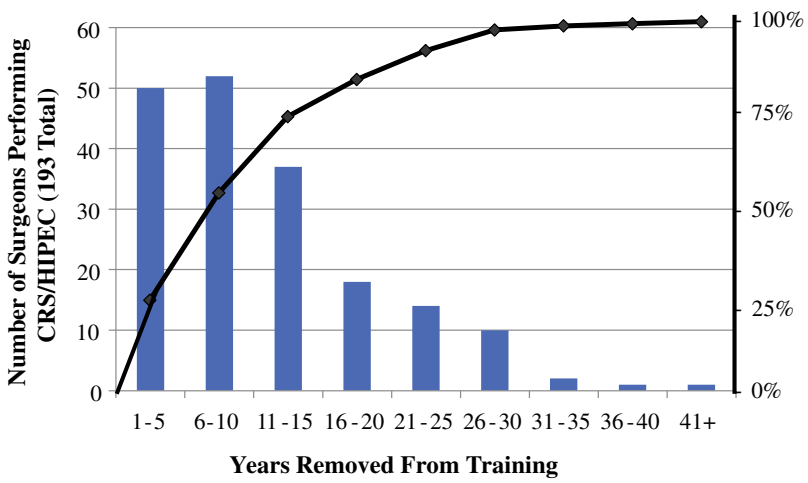

FIG. 3 Surgeons performing CRS/HIPEC by years removed from highest training. Percentages and line graph reflect the cumulative sum of surgeons that are at or below the associated years removed from highest training as a proportion of the total number of surgeons performing CRS/HIPEC. CRS cytoreductive surgery. HIPEC Heated intraperitoneal chemotherapy
South Dakota, Vermont, West Virginia, and Wyoming. Some states do not have surgeons that perform CRS/HIPEC. CRS, cytoreductive surgery. HIPEC Hyperthermic intraperitoneal intraperitoneal chemotherapy

CRS/HIPEC surgeons, 50 (26\%) have 5 years of independent clinical experience or less, and $35(18 \%)$ have 3 years of clinical experience or less, with $51 \%$ of these surgeons not having a senior partner listed at their institution who also performs CRS/HIPEC (Fig. 4). Among the surgeons with 5 years of independent experience or less, the pattern of specialty training follows that of the larger group, with the majority being fellowship-trained in surgical oncology $(80 \%)$, followed by gynecologic oncology $(10 \%)$. Of these 50 surgeons, $80 \%$ are at academic medical centers, $12 \%$ at community centers, and $8 \%$ at academically affiliated centers.

\section{Institutional and Individual Learning Curve with Experience}

The operative inflection point for institutional proficiency and lower complications in performing CRS/HIPEC varies from 130 to 220 cases, with a mean of 168 cases from four studies. ${ }^{9-12}$ These data suggest that for a center managing $10 \mathrm{CRS} / \mathrm{HIPEC}$ cases per year, it would take almost 17 years to meet this mark, and at 20 cases per year, it would take 8.5 years (Fig. 5). In 2016, the average volume of CRS/HIPEC at 12 selected high-volume centers worldwide was estimated to be 55 cases per year (range, 24-123 cases/year). ${ }^{7}$ At that level of volume, it would take 
FIG. 4 Experience as determined by time removed from highest training. The smaller pie chart represents those $\leq 3$ years removed from training and whether or not they have a senior partner that also performs CRS/HIPEC. $C R S$ Cytoreductive surgery. HIPEC Heated intraperitoneal chemotherapy

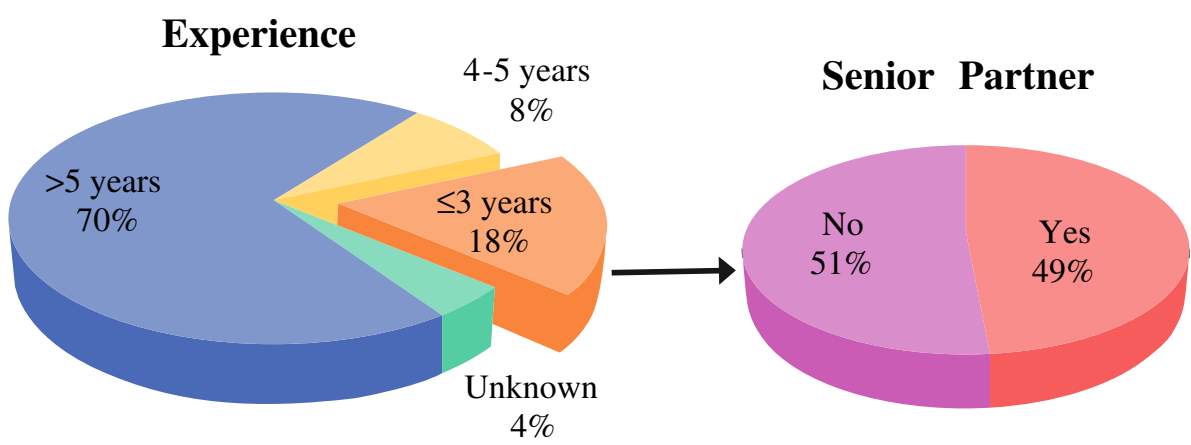

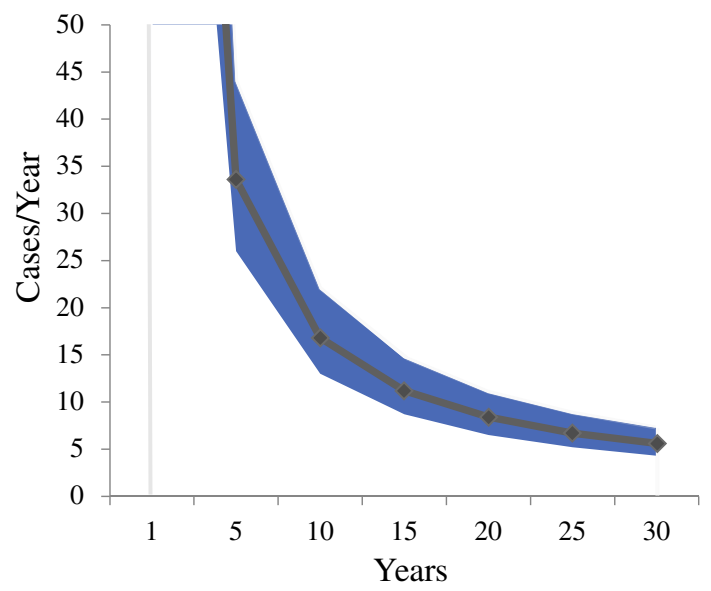

FIG. 5 Time needed to meet the operative inflection point measured by cases per year. The shaded area reflects the reported range in the literature, and the dark gray line represents the mean number of cases necessary to meet the operative inflection point

3 years to meet the operative inflection for proficiency. For new centers, if the proficiency benchmarks are expected to be met by 5 years, it would require 34 cases per year to meet this goal. Of the aforementioned 12 high-volume sites, $3(25 \%)$ would not be able to meet this mark.

\section{DISCUSSION}

The complexity of CRS/HIPEC is well-established and comparable with that of other complex oncologic operations such as pancreaticoduodenectomy, esophagectomy, and major hepatectomy. ${ }^{13}$ Furthermore, CRS/HIPEC is resource-intensive and requires a multidisciplinary team to support both the technical aspects of the procedure (use of intraoperative chemotherapy) and the needs of patients as they recover and face potential long-term sequelae of peritoneal metastases (malignant bowel obstructions and progressive disease).

Despite this, we found that a substantial number of surgeons $(18 \%)$ were removed from training 3 years or less, with half of them having no senior partner who also performs CRS/HIPEC. With the operative inflection point for proficiency and lower complications shown to be about 168 cases, ${ }^{9-12}$ it is unreasonable to expect a single surgeon with no senior partner to meet this metric within 10 years while also managing the other complexities of patient care. Indeed, even at select high-volume centers, $25 \%$ of the CRS/HIPEC surgeons would not be able to meet this quality metric in 5 years. ${ }^{7}$ Furthermore, the survival benefit of CRS depends on the surgeon's ability to perform a complete cytoreduction, ${ }^{6,16-19}$ which findings show to be more likely after an initial learning curve has been met. $^{10-12}$

Additionally, exposure to CRS/HIPEC varies among training programs. No case requirements exist for cytoreduction or administration of intraperitoneal chemotherapy during general surgery residency, nor are these cases required for completion of a surgical oncology o rgynecologic oncology fellowship, which perhaps is more concerning because these are the fellowships that most surgeons performing CRS/HIPEC have completed. ${ }^{20-22}$ Therefore, given the complexity of CRS/HIPEC and the reasonably high percentage of surgeons performing these procedures in the beginning stages of their career, formal training standards should be built into fellowships. For those seeking to start a new program without significant experience in CRS/HIPEC, a formal mentorship effort to support new programs and surgeons should be established. Findings have shown that such mentorship efforts can reduce the learning curve for CRS/HIPEC as it pertains to procedure-related morbidity, mortality, and incomplete cytoreduction $^{23}$ and would help facilitate further growth in the field in a safe and regulated manner.

Although this study helps to illustrate the workforce currently performing CRS/HIPEC, our analysis had limitations. First, although our Internet search was exhaustive, we likely did not capture $100 \%$ of the surgeons performing CRS/HIPEC in the United States. Surgeons who perform CRS/HIPEC might not advertise that they do these procedures and may be missed. Additionally, some surgeons may perform CRS but not HIPEC and thus be missed under the search heading of HIPEC. Although no data specific to CRS/HIPEC exist, given the complexity and morbidity 
associated with these procedures, it could be extrapolated from data regarding similarly complex oncologic operations that these procedures should be centralized to highvolume centers and/or surgeons with considerable expertise on that area. ${ }^{24-26}$

Second, time from completion of training was used as a surrogate for experience. However, not all training experiences are equal, and some surgeons may have had more exposure to CRS/HIPEC during that time than others.

Finally, we were unable to determine how many CRS/ HIPEC cases each surgeon had performed and, given that no current analyses detail the surgical workforce performing pancreaticoduodenectomy, major hepatectomy, or esophagectomy, a direct comparison between the surgical workforce performing CRS/HIPEC and the workforce performing similarly complex oncologic procedures was not possible.

In conclusion, the majority of surgeons performing CRS/HIPEC in the United States are trained in surgical oncology and completed their training 10 or more years ago, with a substantial number in their first 3 years of practice. Although this likely reflects an increased interest in the field, it also highlights the relative inexperience of many surgeons performing these complex procedures. As such, formal training standards and the development of support and mentorship for new programs and surgeons performing CRS/HIPEC should be established.

ACKNOWLEDGMENT The authors recognize both Dr. Mitchell C. Posner and Dr. Jeffrey B. Matthews for their critical review and contribution to the final manuscript.

DISCLOSURE The authors declare that they have no conflict of interest.

\section{REFERENCES}

1. Jayne DG, Fook S, Loi C, et al. Peritoneal carcinomatosis from colorectal cancer. Br J Surg. 2002;89:1545-50.

2. Franko J, Shi Q, Goldman CD, et al. Treatment of colorectal peritoneal carcinomatosis with systemic chemotherapy: a pooled analysis of north central cancer treatment group phase III trials N9741 and N9841. J Clin Oncol. 2012;30:263-7.

3. Thomassen I, van Gestel YR, van Ramshorst B, et al. Peritoneal carcinomatosis of gastric origin: a population-based study on incidence, survival and risk factors. Int $J$ Cancer. 2014;134:622-8.

4. Tseng J, Bryan DS, Poli E, et al. Under-representation of peritoneal metastases in published clinical trials of metastatic colorectal cancer. Lancet Oncol. 2017;18:711-2.

5. van Driel WJ, Koole SN, Sikorska K, et al. Hyperthermic intraperitoneal chemotherapy in ovarian cancer. $N$ Engl J Med. 2018;378:230-40.

6. Verwaal VJ, Bruin S, Boot $\mathrm{H}$, et al. 8-Year follow-up of randomized trial: cytoreduction and hyperthermic intraperitoneal chemotherapy versus systemic chemotherapy in patients with peritoneal carcinomatosis of colorectal cancer. Ann Surg Oncol. 2008;15:2426-32.

7. Rajeev R, Klooster B, Turaga KK. Impact of surgical volume of centers on postoperative outcomes from cytoreductive surgery and hyperthermic intraperitoneal chemoperfusion. J Gastrointest Oncol. 2016;7:122-8.

8. Morano WF, Khalili M, Chi DS, et al. Clinical studies in CRS and HIPEC: trials, tribulations, and future directions: a systematic review. J Surg Oncol. 2018;117:245-59.

9. Kusamura S, Baratti D, Deraco M. Multidimensional analysis of the learning curve for cytoreductive surgery and hyperthermic intraperitoneal chemotherapy in peritoneal surface malignancies. Ann Surg. 2012;255:348-56.

10. Andreasson H, Lorant T, Pahlman L, et al. Cytoreductive surgery plus perioperative intraperitoneal chemotherapy in pseudomyxoma peritonei: aspects of the learning curve. Eur J Surg Oncol. 2014;40:930-6.

11. Polanco PM, Ding Y, Knox JM, et al. Institutional learning curve of cytoreductive surgery and hyperthermic intraperitoneal chemoperfusion for peritoneal malignancies. Ann Surg Oncol. 2015;22:1673-9.

12. Smeenk RM, Verwaal VJ, Zoetmulder FA. Learning curve of combined modality treatment in peritoneal surface disease. $\mathrm{Br} \mathrm{J}$ Surg. 2007;94:1408-14.

13. Foster JM, Sleightholm R, Patel A, et al. Morbidity and mortality rates following cytoreductive surgery combined with hyperthermic intraperitoneal chemotherapy compared with other high-risk surgical oncology procedures. JAMA Netw Open. 2019;2:e186847.

14. United States Census Bureau. United States Summary: 2010 Population and Housing Unit Counts, 2010 Census of Population and Housing. September, 2012.

15. United States Census Bureau. 2018 National and State Population Estimates. 2018. Accessed 18 March 2019.

16. Smeenk RM, Verwaal VJ, Antonini N, et al. Survival analysis of pseudomyxoma peritonei patients treated by cytoreductive surgery and hyperthermic intraperitoneal chemotherapy. Ann Surg. 2007;245:104-9.

17. Coccolini F, Catena F, Glehen O, et al. Complete versus incomplete cytoreduction in peritoneal carcinosis from gastric cancer, with consideration to PCI cut-off: systematic review and meta-analysis. Eur J Surg Oncol. 2015;41:911-9.

18. Winter WE III, Maxwell GL, Tian C, et al. Prognostic factors for stage III epithelial ovarian cancer: a gynecologic oncology group study. J Clin Oncol. 2007;25:3621-7.

19. du Bois A, Reuss A, Pujade-Lauraine E, et al. Role of surgical outcome as prognostic factor in advanced epithelial ovarian cancer: a combined exploratory analysis of 3 prospectively randomized phase 3 multicenter trials: by the Arbeitsgemeinschaft Gynaekologische Onkologie Studiengruppe Ovarialkarzinom (AGO-OVAR) and the Groupe d'Investigateurs Nationaux Pour les Etudes des Cancers de l'Ovaire (GINECO). Cancer. 2009; 115:1234-44.

20. Accreditation Council for Graduate Medical Education. Defined Category Minimum Numbers for General Surgery Residents and Credit Role 2019. https://www.acgme.org/Portals/0/DefinedCate goryMinimumNumbersforGeneralSurgeryResidentsandCreditRol e.pdf. Accessed 28 March 2019.

21. Accreditation Council for Graduate Medical Education. Case minimum requirements: complex general surgical oncology 2016. https://www.acgme.org/Portals/0/Defined_Category_Mini mum_Numbers_Complex_General_Surgical_Oncology.pdf. Accessed 28 April 2019.

22. The American Board of Surgery. Booklet of Information Surgery. 2018. http://www.absurgery.org/xfer/BookletofInfo-Surgery.pdf. Accessed 28 April 2019. 
23. Kusamura S, Baratti D, Virzi S, et al. Learning curve for cytoreductive surgery and hyperthermic intraperitoneal chemotherapy in peritoneal surface malignancies: analysis of two centres. J Surg Oncol. 2013;107:312-9.

24. Harmon JW, Gordon TA, Bowman HM, et al. Hospital volume can serve as a surrogate for surgeon volume for achieving excellent outcomes in colorectal resection. Ann Surg. 1999;230:404-11.

25. Begg CB, Hoskins WJ, Brennan MF. Impact of hospital volume on operative mortality for major cancer surgery. JAMA. 1998;280:1747-51.
26. Chowdhury MM, Dagash H, Pierro A. A systematic review of the impact of volume of surgery and specialization on patient outcome. Br J Surg. 2007;94:145-61.

Publisher's Note Springer Nature remains neutral with regard to jurisdictional claims in published maps and institutional affiliations. 